\title{
Eigenmode-based Capacitance Calculations with Applications in Passivation Layer Design
}

\author{
Thomas Demeester and Daniël De Zutter, Fellow, IEEE
}

\begin{abstract}
The design of high-speed metallic interconnects such as microstrips requires the correct characterization of both the conductors and the surrounding dielectric environment, in order to accurately predict their propagation characteristics. A fast boundary integral equation approach is obtained by modeling all materials as equivalent surface charge densities in free space. The capacitive behavior of a finite dielectric environment can then be determined by means of a transformation matrix, relating these charge densities to the boundary value of the electric potential. In this paper a new calculation method is presented for the important case that the dielectric environment is composed of homogeneous rectangles. The method, based on a surface charge expansion in terms of the Robin eigenfunctions of the considered rectangles, is not only more efficient than traditional methods, but also more accurate, as shown in some numerical experiments. As an application, the design and behavior of a microstrip passivation layer is treated in some detail.
\end{abstract}

Index Terms-Interconnects, capacitance, Robin eigenfunctions, passivation layer, microstrip design.

\section{INTRODUCTION}

$\mathbf{H}$ IGH speed interconnects both on-board and on-chip that display substantial wave effects, are often long enough to be modeled as two-dimensional transmission line structures, such as microstrips or striplines, both on the printed circuit board (PCB) level, as on-chip. For such models, the so-called 'RLGC' circuit matrices, i.e., R, L, G, C, respectively, the resistance, inductance, conductance, and capacitance matrices, in combination with the telegrapher's equations describe the behavior of the fundamental propagation modes along the lines. It is shown in [1] that for typical high-speed interconnect applications, the so-called quasi transverse magnetic (quasiTM) conditions remain valid even for the highest operating frequencies. This is the case, as long as the corresponding longitudinal wavelength remains enough longer than the transverse dimension over which the fields are relevant. If this were not the case, the structure would no longer be apt for an efficient data transmission. Full-wave solutions to Maxwell's equations as a basis for the RLGC matrix extraction have been proposed as well [2]. These however lead to different models (which all coincide in the quasi-TM frequency range), and they loose a major advantage of quasi-TM models: the quasi-TM conditions lead to a decoupling of the total electromagnetic field problem into (i) a quasi-static complex capacitance problem (to determine the complex capacitance $\mathrm{C}+\mathrm{G} / j \omega$, with $\omega$ the angular frequency) and (ii) a quasi-TM complex inductance problem (to calculate $\mathrm{L}+\mathrm{R} / j \omega$ ).

The authors are with the Department of Information Technology, Ghent, Sint-Pietersnieuwstraat 41, B-9000 Gent, Belgium. Tel: +32 9264 33 27, Fax: +329264 35 93. E-mail: thomas.demeester@intec.ugent.be, daniel.dezutter@intec.ugent.be.
The numerical solution of a capacitance problem has been under investigation for a long time now and many different solution methods have been developed. Some of these, as well as a further overview of the existing literature, are found in [3][11]. Even today, the quasi-static approach to determine the capacitive behavior of many practical interconnects remains valid.

The current paper proposes a new numerical solution method that is similar to the recent technique described in [1], in the sense that it allows for an efficient boundary integral equation based solution. The considered configurations are not restricted to infinitely wide layered media but require that the cross-section can be divided into an ensemble of homogeneous rectangles, which is often the case. As remarked by one of the reviewers, a solution technique using a standard surface integral equation for the conductor-dielectric electrostatic problem, see e.g. [12] section 3.12.1, is a valid alternative for the technique presented in this paper and can be applied to arbitrary shapes. An example of the use of this technique can be found in [13]. In our technique the use of the more singular derivative of the Green's function is avoided.

In section II, some elements of the method of [1] are briefly summarized, and from these the new method is developed, with a mathematical description in Section III. In the numerical examples of Section IV, firstly some properties of the new field expansion functions are illustrated, secondly a comparison between the method of [1] and the new technique is made, and finally, a passivation layer design example is discussed, as the new method appears to be well-suited for thin layers.

\section{Capacitance Calculations}

For conductors in a lossy dielectric environment that exists of finite homogeneous subregions, a fast solution for the capacitance problem, based on a boundary integral equation, was presented in [1]. Some aspects of this method are briefly recapitulated here, as they are needed in the sequel. First, all materials are replaced by equivalent surface charge densities $\rho$ in free space, such that the quasi-static 2-D Green's function $G_{0}$ of free space can be used to relate the electric potential $\phi$ to these charges, according to

$$
\phi(\boldsymbol{r})=-\int_{\forall c_{i}} G_{0}\left(\boldsymbol{r} \mid \boldsymbol{r}^{\prime}\right) \rho\left(\boldsymbol{r}^{\prime}\right) d c\left(\boldsymbol{r}^{\prime}\right)
$$

in which the integral runs over the boundary $c_{i}$ of each homogeneous subregion $i$ and with

$$
G_{0}\left(\boldsymbol{r} \mid \boldsymbol{r}^{\prime}\right)=\frac{1}{4 \pi \epsilon_{0}} \ln \left(\left|\boldsymbol{r}-\boldsymbol{r}^{\prime}\right|\right) .
$$


Now, $\rho$ and $\phi$ are discretized as

$$
\rho(\boldsymbol{r}) \approx \sum_{m} R_{m} b_{m}(\boldsymbol{r}) \text { and } \phi(\boldsymbol{r}) \approx \sum_{m} \Phi_{m} b_{m}(\boldsymbol{r})
$$

for $\boldsymbol{r}$ on the considered boundary, and $\left\{b_{m}\right\}$ a set of appropriate basis functions. A Galerkin weighing of (1), evaluated on the material boundaries, yields a first set of relationships between the unknowns $R_{m}$ and $\Phi_{m}$, in the form

$$
\left[\begin{array}{l}
\mathbf{V}_{\mathbf{c}} \\
\boldsymbol{\Phi}_{\mathbf{d}}
\end{array}\right]=-\left[\begin{array}{ll}
\mathrm{G}_{\mathrm{cc}} & \mathrm{G}_{\mathrm{cd}} \\
\mathrm{G}_{\mathrm{dc}} & \mathrm{G}_{\mathrm{dd}}
\end{array}\right] \cdot\left[\begin{array}{l}
\mathbf{R}_{\mathbf{c}} \\
\mathbf{R}_{\mathbf{d}}
\end{array}\right]
$$

in which the expansion coefficients for the charge, respectively, electric potential are taken together for the conductors in $\mathbf{R}_{\mathbf{c}}$ and $\mathbf{V}_{\mathbf{c}}$, and for the dielectrics in $\mathbf{R}_{\mathbf{d}}$ and $\boldsymbol{\Phi}_{\mathbf{d}}$. Furthermore, the constant voltage $\mathbf{V}_{\mathbf{c}}$ on the conductors' boundaries is used as excitation, and the equivalent charges on the boundary of dielectric subregion $i$ can be related to the boundary value of its potential, through

$$
\begin{aligned}
\rho(\boldsymbol{r}) & =\left(\epsilon_{i}-\epsilon_{0}\right) \frac{\partial \phi(\boldsymbol{r})}{\partial n}, \quad \boldsymbol{r} \in c_{i} \\
& =\left(\epsilon_{i}-\epsilon_{0}\right) \oint_{c_{i}} \mathcal{D}\left(\boldsymbol{r}, \boldsymbol{r}^{\prime}\right) \phi\left(\boldsymbol{r}^{\prime}\right) d c\left(\boldsymbol{r}^{\prime}\right),
\end{aligned}
$$

with $\mathcal{D}$ the Dirichlet-to-Neumann operator (DtN), mapping $\phi_{c_{i}}$ onto its normal derivative along $c_{i}$, and with $\epsilon_{i}$ the permittivity of material $i$. In its discretized form, and written for all dielectrics together, (6) becomes

$$
\mathbf{R}_{\mathrm{d}}=\mathrm{D} \boldsymbol{\Phi}_{\mathrm{d}}
$$

which allows, upon substitution into (4), to determine all unknown coefficients. The total charge on each conductor, leads to the entries of the capacitance (and conductance) matrix if each conductor is set in turn to $1 \mathrm{~V}$ while the others are kept on $0 \mathrm{~V}$.

The method as explained above works very well, but has one major drawback due to the discretization of the DtN operator into the DtN matrix D. The calculation of $D$ in the quasi-static frequency range is based on an expansion of $\phi$ over the dielectric subregions (discretized in rectangles or triangles) in terms of sine basis functions on each side, while enforcing the exact solution for $\phi$ which, in the quasi-TM limit, satisfies Laplace's equation $\nabla^{2} \phi(x, y)=0$. Its normal derivative is then determined as the superposition of the normal derivatives of each basis function. This is described in [14] and [15], for rectangular, respectively, triangular geometries, but in this paper we focus on the important case of rectangular dielectric subregions. A Galerkin weighing procedure yields an expansion of $\phi$ with a minimal quadratic error along the boundary. The small components in $\phi$, which vary rapidly along the boundary, get a more important weight in $\partial \phi / \partial n$. On the one hand, enough higher harmonics are required for the representation of 'any' boundary value of $\phi$, but on the other hand, a small error on these components leads to a strongly increased error in the normal derivative, as demonstrated further on. It especially deteriorates the accuracy of $\rho$ near the corners, because of an often imperfect estimation of $\phi$ at the corner points, used to eliminate the Gibb's effect [14].
In Section III, this problem is solved by means of an expansion with a minimal quadratic error for $\rho$ instead of $\phi$ over the considered dielectric regions. This has as a direct consequence that the capacitance calculations, by integrating $\rho$, are much more accurate. Indirectly, the error on $\phi$, in turn no longer minimal in a quadratic sense, remains small as we integrate $\rho$ to obtain $\phi$, and because $\phi$ is generally much smoother than $\rho$. Of course, the boundary value of $\phi$ on a dielectric block cannot be determined unambiguously from $\partial \phi / \partial n$. Therefore, we will first extract a constant $\phi_{0}$ from $\phi$, by averaging over the boundary. For the remaining component $\left(\phi-\phi_{0}\right)$, its relation to $\partial \phi / \partial n$ is bijective, and we will construct an operator $\mathcal{B}$ which maps $\partial \phi / \partial n$ on boundary $c_{i}$ onto $\left(\phi-\phi_{0}\right)$, such that with (5),

$$
\phi(\boldsymbol{r})=\phi_{0}+\oint_{c_{i}} \frac{\mathcal{B}\left(\boldsymbol{r}, \boldsymbol{r}^{\prime}\right)}{\epsilon_{i}-\epsilon_{0}} \rho\left(\boldsymbol{r}^{\prime}\right) d c\left(\boldsymbol{r}^{\prime}\right), \quad \boldsymbol{r} \in c_{i} .
$$

The discretized form of (8), taken together for all dielectrics, becomes

$$
\Phi_{\mathrm{d}}=\boldsymbol{\Phi}_{0}+\mathrm{B} \mathbf{R}_{\mathrm{d}}
$$

in which $\boldsymbol{\Phi}_{\mathbf{0}}$ is a column vector, for each dielectric $i$ containing a different unknown constant potential $\phi_{0, i}$. Substitution of (9) into (4) leads to a system of equations to be solved with respect to the unknown $\mathbf{R}_{\mathbf{c}}, \mathbf{R}_{\mathbf{d}}$ and the mean dielectric boundary potentials $\phi_{0, i}$. This system has to be completed with as many extra equations as there are unknowns $\phi_{0, i}$, expressing the requirement that the total equivalent charge on each dielectric has to remain zero.

\section{ROBIN EIGENFUNCTION EXPANSION OF THE CHARGE DENSITIES}

In this section, we will explain how the relation

$$
\phi(\boldsymbol{r})-\phi_{0}=\oint_{c} \mathcal{B}\left(\boldsymbol{r}, \boldsymbol{r}^{\prime}\right) \frac{\partial \phi\left(\boldsymbol{r}^{\prime}\right)}{\partial n} d c\left(\boldsymbol{r}^{\prime}\right), \quad \boldsymbol{r} \in c
$$

is discretized along the boundary $c$ of a rectangular area $S \leftrightarrow\left\{-x_{0} \leq x \leq x_{0},-y_{0} \leq y \leq y_{0}\right\}$, for a quantity $\phi$ satisfying Laplace's equation inside $S$. We will need the following expansion

$$
\frac{\partial \phi(\boldsymbol{r})}{\partial n}=\sum_{n=1}^{N} \chi_{n} \psi_{n}(\boldsymbol{r}), \quad \boldsymbol{r} \in c
$$

with $N$ high enough (see Section IV), and in which the basis functions $\psi_{n}$ satisfy

$$
\begin{aligned}
\nabla^{2} \psi_{n}(\boldsymbol{r}) & =0, \quad \boldsymbol{r} \in S \\
\frac{\partial \psi_{n}(\boldsymbol{r})}{\partial n} & =\lambda_{n} \psi_{n}(\boldsymbol{r}), \quad \boldsymbol{r} \in c \\
\oint_{c} \psi_{n}(\boldsymbol{r}) \psi_{m}(\boldsymbol{r}) d c & =\delta_{n m}
\end{aligned}
$$

with $\delta_{n m}=1$ if $n=m$, and else $\delta_{n m}=0$. Because of the socalled Robin boundary condition (13), the orthonormal set of eigenfunctions we wish to construct, are Robin eigenfunctions of $S$. Separation of the variables (SoV) in this case (omitting the index $n$ ), and already taking into account (13) on the sides 
$x=0$ and $y=0$, yields the following four independent sets of solutions

$$
\psi(x, y)=C\left\{\begin{array}{c}
\cos \gamma x \\
\sin \gamma x
\end{array}\right\} \cdot\left\{\begin{array}{c}
\cosh \gamma y \\
\sinh \gamma y
\end{array}\right\}
$$

with a normalization coefficient $C$ yet to be determined. The four equations are obtained by combining each of the two terms between the first brackets with each of the terms between the second brackets. The remaining conditions,

$$
0=\left[\frac{\partial \psi}{\partial x}-\lambda \psi\right]_{x=x_{0}} \text { and } 0=\left[\frac{\partial \psi}{\partial y}-\lambda \psi\right]_{y=y_{0}}
$$

lead with (15), and by elimination of $\lambda$, to

$$
\left\{\begin{array}{c}
-\tan \gamma x_{0} \\
\cot \gamma x_{0}
\end{array}\right\}=\left\{\begin{array}{c}
\tanh \gamma y_{0} \\
\operatorname{coth} \gamma y_{0}
\end{array}\right\}
$$

displaying four different equations, corresponding to the different combinations for $\psi$ in (15). Four sets of solutions for $\gamma$ can now be calculated, which are all real and positive (as the corresponding negative solutions lead to the same eigenfunctions and are thus omitted), with the corresponding eigenfunction given by (15) and the eigenvalues by

$$
\lambda=\gamma\left\{\begin{array}{c}
-\tan \gamma x_{0} \\
\cot \gamma x_{0}
\end{array}\right\} .
$$

We see that, if we replace $\gamma$ in (17) by $j \gamma$, the same eigenvalue equations are found, provided $x_{0}$ and $y_{0}$ are exchanged. The same observation holds for (15). As a result, the algorithm to solve (17) can be used again, with $x_{0}$ and $y_{0}$ exchanged. The real solutions one finds now, are in fact the remaining strictly imaginary solutions of (17). The numerical procedure to solve (17) is straightforward. For the examples treated in this paper, the secant method is used, which converges rapidly and to an arbitrary precision, due to the regularity in the zeros, and the asymptotic behavior for large values of $\gamma$.

Combining (12) and (13) with Gauss' law, shows that two eigenfunctions $\psi_{i}$ and $\psi_{j}$ are always orthogonal with respect to integration over $c$ if they have different eigenvalues $\lambda_{i} \neq \lambda_{j}$. In some cases, different functions are found with the same eigenvalues, e.g., for $x_{0}=y_{0}$, but in these cases those functions are orthogonal as well. A more rigorous mathematical analysis would be outside the scope of this paper, however. Finally, for each eigenfunction $\psi_{i}$, the normalization coefficient $C_{i}$ can be determined by equating $\psi_{i}^{2}$, after integration over $c$, to one. As an illustration, the boundary value of a few Robin eigenfunctions is shown in Fig. 1, for a rectangle with dimensions $2 \times 1$, with consecutive sides, respectively, $c_{1}, c_{2}$, $c_{3}$, and $c_{4}$. The eigenvalues are given as well. For the higher values of $\gamma_{n},(18)$ shows that $\lambda \approx \gamma$, and from (17) it then follows that $\gamma x_{0} \approx(2 k+1) \pi / 4, k \in \mathbb{Z}$ (as is the case for $\left.\psi_{22}\right)$, or, for the second set of eigenvalues, $\gamma y_{0} \approx(2 k+1) \pi / 4$ (as for $\psi_{20}$ and $\psi_{21}$ ). Beyond the first 20 eigenvalues, these approximations form a very good starting guess in the root finding procedure, allowing to reach a sufficient accuracy within very few iterations. Notice the presence of the constant eigenfunction $\psi_{0}$, corresponding to the zero eigenvalue. $\psi_{0}$ is
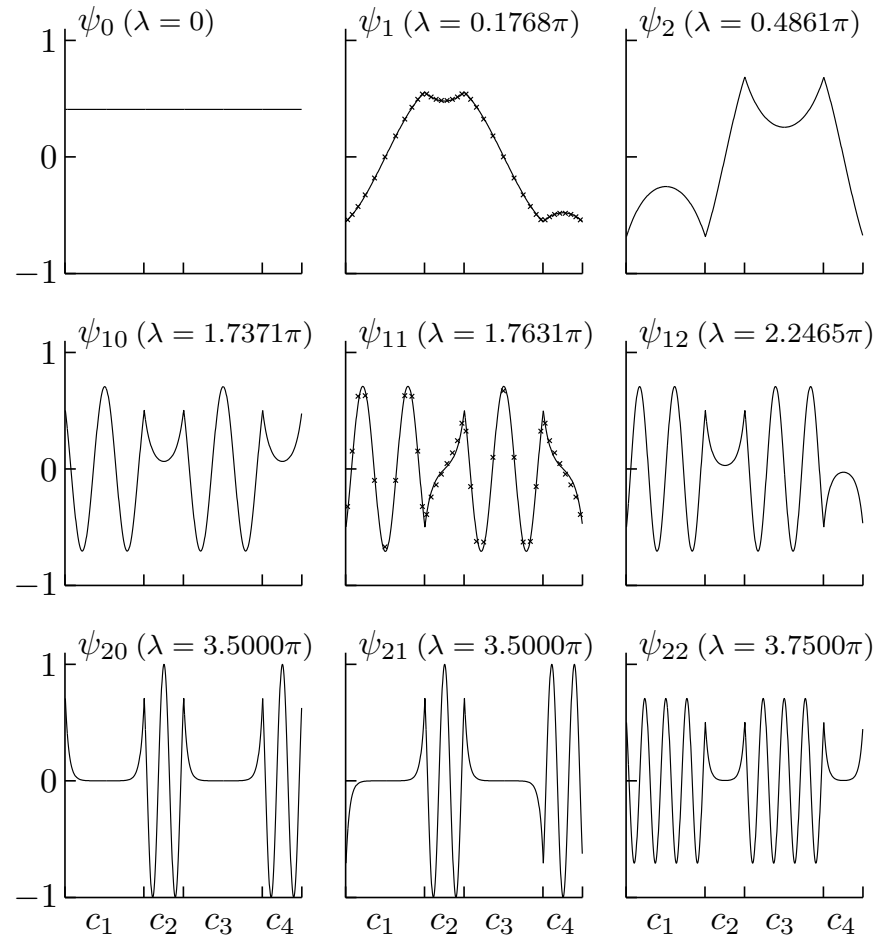

Fig. 1: Boundary value of some Robin eigenfunctions, for a rectangle with dimensions $2 \times 1$.

not present in (11), as the mean of $\partial \phi / \partial n$ has to be zero, but in a general expansion it is of course required.

Consider again the expansion (11), in which $\partial \phi / \partial n$ is discretized as

$$
\frac{\partial \phi(\boldsymbol{r})}{\partial n}=\sum_{m} E_{m} b_{m}(\boldsymbol{r}), \quad \boldsymbol{r} \in c
$$

For the basis functions $b_{m}$ along $c$, pulses are used in the examples in Section IV, but other choices are equally possible. The Galerkin weighing procedure leads from (11) and (19) to the discretized form

$$
X=P^{\top} E
$$

in wich $\mathrm{X}$ and $\mathrm{E}$ contain the coefficients $\chi_{n}$ (see (11)), respectively, $E_{m}$, and

$$
[\mathrm{P}]_{m n}=\oint_{c} b_{m}(\boldsymbol{r}) \psi_{n}(\boldsymbol{r}) d c .
$$

If we expand $\phi$ on $S$ in terms of the new eigenfunctions,

$$
\phi(\boldsymbol{r})=\sum_{n=0}^{N} \alpha_{n} \psi_{n}(\boldsymbol{r}), \quad \boldsymbol{r} \in S
$$

we find that

$$
\alpha_{0} \psi_{0}=\frac{1}{\mathcal{C}} \oint_{c} \phi(\boldsymbol{r}) d c(\boldsymbol{r}) \stackrel{\text { def }}{=} \phi_{0}
$$

with $\mathcal{C}$ the perimeter of $S$. Comparing the normal derivative of (22) to (11), shows with (23) that

$$
\phi(\boldsymbol{r})-\phi_{0}=\sum_{n=1}^{N} \frac{\chi_{n}}{\lambda_{n}} \psi_{n}(\boldsymbol{r}), \quad \boldsymbol{r} \in c
$$


and if we define $\Delta \Phi_{m} \stackrel{\text { def }}{=} \Phi_{m}-\phi_{0}$, and weigh (24) and (3) with each of the basis functions $b_{m}$, we find the direct discretization of (10),

$$
\Delta \Phi=\mathrm{BE}, \quad \text { with } \mathrm{B}=\mathrm{T}^{-1} \mathrm{P} \wedge^{-1} \mathrm{P}^{\top}
$$

in which the column vector $\Delta \Phi$ contains the coefficients $\Delta \Phi_{m}$, the diagonal matrix $\wedge$ contains the eigenvalues $\lambda_{n},(n=1, \ldots, N)$ on its diagonal, and

$$
[\mathrm{T}]_{m \tilde{m}}=\oint_{c} b_{m}(\boldsymbol{r}) b_{\tilde{m}}(\boldsymbol{r}) d c(\boldsymbol{r}) .
$$

For the calculation of the DtN matrix D as in [14], analogous matrices as $\mathrm{P}$ and $\mathrm{P}^{\top}$ are required, but instead of the diagonal matrix $\Lambda^{-1}$ in (25) a much slower matrix multiplication with a large non-sparse matrix is required, to transform the sine expansion into the corresponding normal derivative. Here a numerical root finding procedure is required to determine the diagonal elements of $\Lambda$. Globally, the determination of $B$ remains more efficient, apart from the fact that the results are by far more accurate (see Section IV-B).

It is worth mentioning that these Robin eigenfunctions represent a very natural basis to calculate the matrix B from. The reason is, that its eigenvectors are a discretized version of the eigenfunctions $\psi_{n}$, with eigenvalues approximately $1 / \lambda_{n}$. This directly follows from the boundary condition (13) in comparison with the eigenvalue equation for B. As an illustration, the first and eleventh eigenvectors of $B$ are plotted in Fig. 1 with small cross ' $x$ ' symbols, and compared with $\psi_{1}$ and $\psi_{11}$, respectively, where the rectangle was discretized with 38 intervals along its boundary. In order to compensate for the different normalization, they were rescaled with a scalar factor of $1 / \sqrt{\left(\sum_{k} d_{k} V_{k}\right)}$ (with $d_{k}$ the interval width, and $V_{k}$ the entries of the considered eigenvector with unit euclidean norm), and the correspondence is indeed good.

\section{NUMERICAL EXAMPLES}

\section{A. Investigation of the Expansion Properties}

As a first, theoretical, example, the numerical error on $\partial \phi / \partial n$ is compared as obtained from the DtN matrix, with respect to the new expansion in Robin eigenfunctions. Consider again the rectangle $S$ from Section II, this time discretized with 38 intervals along its boundary, as shown in the inset of Figure 2. Take an arbitrary function $\phi(x, y)=$ $\cosh \left(1-x / x_{0}\right) \sin \left(y / x_{0}\right)$ (with $x_{0}=1$ and $\left.y_{0}=0.5\right)$ that satisfies Laplace's equation. In Fig. 2(a), the continuous value of $\partial \phi / \partial n$ is shown as a reference, as well as two pulsebased approximations (displaying the coefficients in the middle of the intervals). These were obtained as follows. From a Galerking weighing of the exact boundary value of $\phi$, the vector $\Phi$ was obtained, and the coefficients displayed with ' $x$ '-symbols, are found as D $\Phi$ from the DtN matrix D. The coefficients shown in dots are found from an expansion in the Robin eigenfunctions, with the same number of basis functions as used to calculate the DtN matrix. From E, the 'best' possible discretization (in a least squares sense) of $\partial \phi / \partial n$, we obtain the coefficients of the Robin functions as $\mathrm{P}^{\top} \mathrm{E}$. Again projecting these on the basis of pulses yields
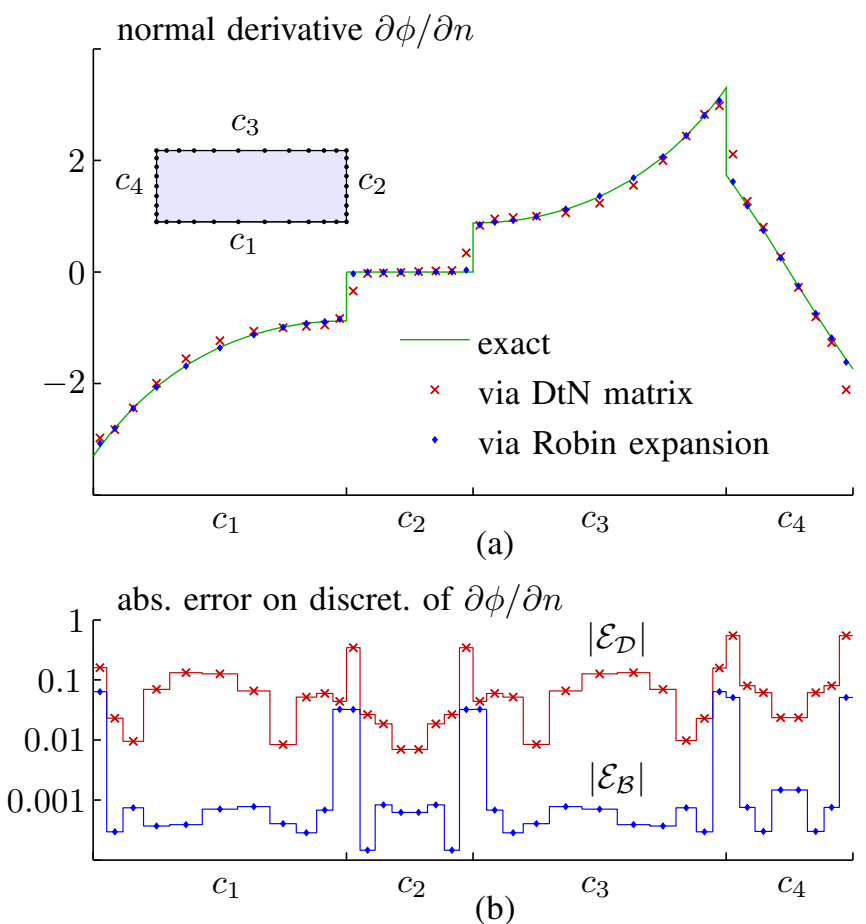

Fig. 2: (a) Boundary value $\phi$ (continuous and discretized) for a rectangle with dimensions $2 \times 1$, (b) normal derivative $\partial \phi / \partial n$ (cont., discret., and approximations via the DtN matrix and via the Robin expansion, both with the same amount of expansion functions), and (b) absolute error on both approximations.

$\mathrm{T}^{-1} \mathrm{PP}^{\top} \mathrm{E}$, shown with dots in Fig. 2. The absolute value of the difference between the ideal coefficients $E$ and both approximations is shown in Fig. 2(b). The overall accuracy using the Robin eigenfunction expansion is, as expected, much better than with the DtN matrix. The reason is primarily due to the inaccurate treatment near the corners, and to the increased weight of higher order contributions in the DtN approach.

In this example, 304 expansion functions were used (to both calculate $\mathrm{D}$ and $\mathrm{P}$ ), or 8 times the number of discretization intervals. Let us in general find out how the error behaves in terms of the number $N$ of basis functions (for Dirichletvs. Robin expansion), and in relation to the number $M$ of discretization intervals. In Fig. 3, the error is shown for varying $N$ (normalized by $M$ ), for two different discretizations, i.e., $M=38$ (as for Fig. 2) and $M=98$. The shown error is a relative root mean square (RMS) error, defined as

$$
\text { error }=\sqrt{\frac{\oint_{c}\left([\partial \phi / \partial n]_{\text {approx. }}-\partial \phi / \partial n\right)^{2} d c}{\oint_{c}(\partial \phi / \partial n)^{2} d c}}
$$

in which pulses are used to discretize the continuous quantities. We observe a minimum in the error for the DtN approach, when about as many expansion functions are used as the number of discretization segments. If more expansion functions are used, the error grows, due to the reinforcement of inaccuracies in the calculation of the normal derivatives. In practice, the boundary value of $\phi$ might vary much faster over the sides 


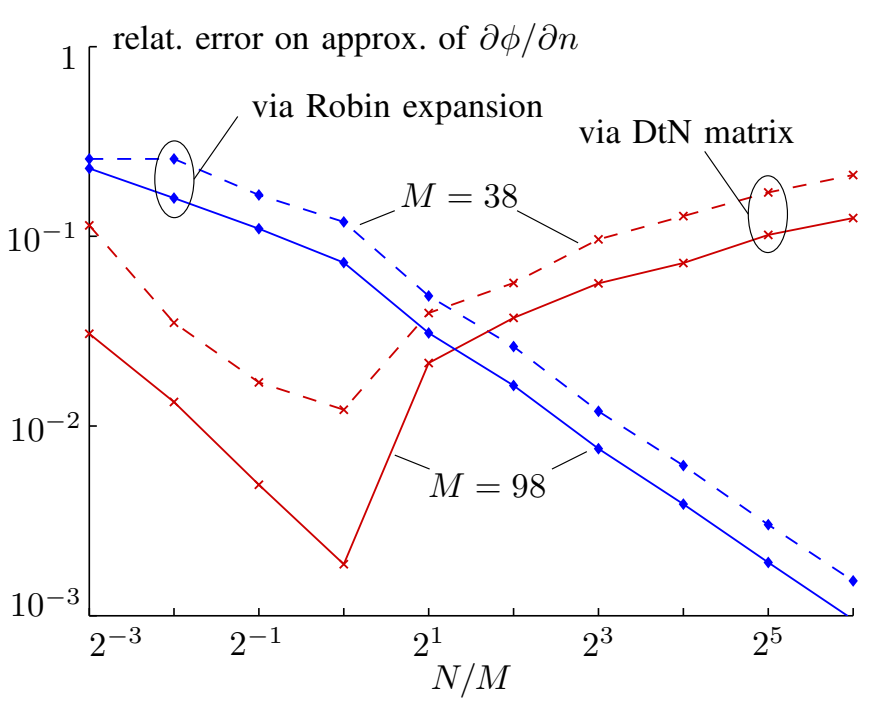

Fig. 3: Relative RMS error on both approximations of $\partial \phi / \partial n$ (via DtN matrix, and via Robin expansion), for the configuration from Fig. 2. $N$ is the number of expansion functions, $M$ the number of discretization intervals.

than in this theoretical example. In that case, a number of sine functions equal to the number of intervals along that side could be insufficient to expand the boundary value, and the error would consequently increase. If too many expansion functions are chosen, the error becomes much larger than the monotonically decreasing error of the Robin expansion (see the results in IV-B). The behavior for a finer discretization is qualitatively the same, but with an overall lower error.

The numerical errors shown in Fig. 2 and Fig. 3 are an incomplete way to compare the accuracy of both methods. The error on $\partial \phi / \partial n$ for the DtN expansion is necessarily larger than for the Robin expansion, as the former one involves a change of basis, subsequently the transformation from $\phi$ to $\partial \phi / \partial n$ and then again a change of basis, whereas the latter expansion is only a cascade of two basis transformations. One could put forward the question how large the error on $\phi$ would be in the Robin case, including the transformation $\partial \phi / \partial n \rightarrow \phi$, but then again, it is not possible to directly compare this error to the ones shown in Fig. 3. Therefore, in the next paragraphs a direct comparison between both methods is performed on the capacitance level.

\section{B. Comparison DtN vs. Robin Method}

Consider the differential pair structure shown in Fig. 4, used in this section without the dielectric coating $(\Delta=0)$, and with $w=6.56$ mils. The capacitance problem is solved by both the DtN method and the new Robin method. To investigate the influence of the discretization and the number of expansion functions used inside the substrate, its width $\mathrm{W}$ is varied from narrow $(W=2 w+s=18.11$ mils $=460 \mu \mathrm{m}$, only underneath the differential pair) up to very wide ( $W=$ 100 mils $=2540 \mu \mathrm{m})$. As a reference, the same structure was simulated with the Capcad software developed by $\mathrm{F}$. Olyslager, which uses a boundary integral equation approach,

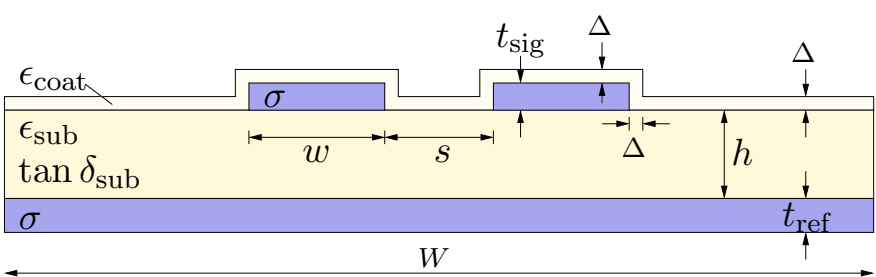

Fig. 4: Coated differential pair (not shown on scale), with $t_{\mathrm{sig}}=0.5 \mathrm{oz}(1 \mathrm{oz}=34.8 \mu \mathrm{m}), t_{\mathrm{ref}}=1 \mathrm{oz}, h=5 \mathrm{mils}$ $(1 \mathrm{mil}=25.4 \mu \mathrm{m}), s=5$ mils, $\sigma=58 \mathrm{MS} / \mathrm{m}$ (copper), $\epsilon_{\text {sub }}=4.3 \epsilon_{0}$ and $\tan \delta_{\text {sub }}=0.02$. Variable parameters are $w, \Delta$ and $\epsilon_{\text {coat }}$.

based on the Method of Moments with the Green's function of an infinitely wide multilayered medium [6]. That program only deals with perfect conductors (including an infinitely wide perfect conducting ground plane), but for the quasi-static capacitance problem, the finite conductivity of the conductors is irrelevant. Nonetheless, this only yields the asymptotic solution for a very wide substrate.

In order to learn as much as possible about the numerical behavior of both methods, simulations were done for three different discretizations. In the configurations designated as 'coarse', 'medium' and 'fine', the number of segments along the width of each conductor are 6, 12 and 24, respectively (and 50 for the reference simulation with Capcad). For each simulation, the discretization is recalculated automatically by our software and hence, the segment width slightly increases for wider substrates. In order to keep the comparison between the different simulations transparant, the discretization per side is chosen to be fairly uniform (whereas normally it would be much denser near corners in order to increase the accuracy for a lower number of unknowns).

For both methods, the parameter $\alpha$ is defined as the number of expansion functions (Dirichlet or Robin) over the substrate, divided by the number of discretization segments along its boundary. The different values of this parameter that are used in the simulations are $\alpha=1,2,4,8$ and 16 . Note that for a finer discretization, a proportionally larger number of basis functions is used for the same $\alpha$. In Fig. 5, the self-capacitance entries $C_{11}$ are displayed. Using a dashed line, the reference value for an infinitely wide substrate is indicated on each figure.

The left column displays the results for the DtN method, with a finer discretization from top to bottom. In each case, the capacitance results increase for a higher $\alpha$. This confirms our assumption that one cannot add too many higher order Dirichlet functions in the expansion of $\phi$, as small errors on $\phi$ lead to unacceptable errors on $\partial \phi / \partial n$. Clearly, the results are better for a finer discretization, and the algorithm is normally used for a fine discretization and $\alpha$ around 3 .

For the Robin method, displayed on the right-hand side, the behavior is different. On the one hand, a factor $\alpha=1$ leads to unreliable results, indicating that the same number of expansion functions as the number of boundary segments is not sufficient to calculate $\phi$ from $\partial \phi / \partial n$. As soon as $\alpha>1$, the results are very consistent, and not only for 


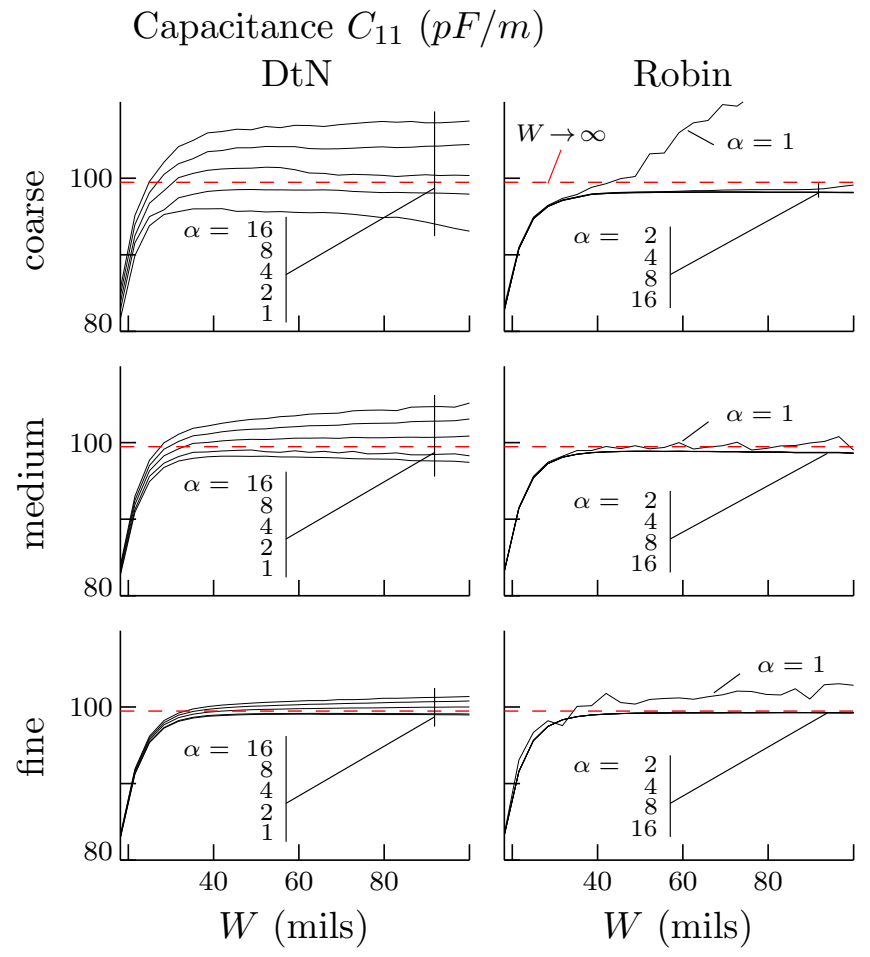

Fig. 5: Capacitance $C_{11}$ for the structure of Fig. 4 (with $\Delta=0, w=6.56$ mils), with the DtN method (left), and the Robin method (right), for 3 different discretizations ('coarse', 'medium' and 'fine'), as a function of the substrate width $W$ and the parameter $\alpha$, the ratio of the number of expansion functions to discretization segments. The dashed line is the reference value for an infinitely wide substrate.

Fig. 6: Simulated geometry, consisting of 13 rectangular blocks (shown on scale, but displaying only half of the simulated substrate width). The dimensions are as in Fig. 4, with $w=6.56$ mils, and $\Delta=0.5 \mathrm{oz}$.

the fine discretization (although in the 'coarse' case, there is a $1.3 \%$ underestimation of the result, due to the inaccurate discretization).

Two major advantages of the Robin method have come forward. On the one hand, the results converge to a fixed value, as soon as sufficient Robin functions are used (which is not the case for the DtN method). On the other hand, the factor $\alpha$ is about the same as for the DtN method, but it allows for a coarser discretization. This means that the absolute number of Robin functions required is lower than the required number of Dirichlet functions, and the total number of unknowns is smaller than for a similar accuracy with the DtN method.

\section{Modeling of a Passivation Layer}

As an application example, we will study the effect of a thin dielectric passivation layer on top of the microstrip structure

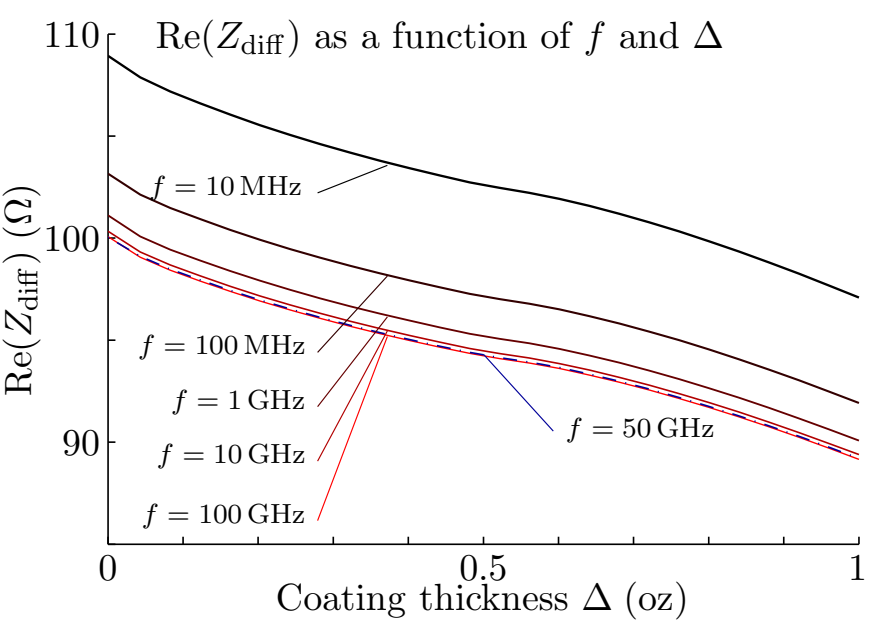

Fig. 7: $\operatorname{Re}\left(Z_{\text {diff }}\right)(\Omega)$ for the structure of Fig. 4, with $w=$ 6.56 mils and $\epsilon_{\text {coat }}=3.3 \epsilon_{0}$, for variable coating thickness $\Delta$, and shown at several frequencies. The dash-dot line at $50 \mathrm{GHz}$ is used as a reference for further simulations.

used in the previous example. Such a coating consists of an inert dielectric material, and serves the purpose of protecting the surface against humidity and corrosion [16]. This however leads to an increased total amount of dielectric material near the traces, and hence a lower characteristic impedance. This effect is often compensated by reducing the trace width, which in turn leads to an increased resistance and hence higher propagation losses.

The new technique developed in this paper to deal with dielectrics, is well-suited for the simulation of a thin passivation layer, due to its accuracy, as seen in Section IV-B. We will explore in detail how the characteristic impedance of a differential microstrip pair is affected by the thickness and dielectric constant of the passivation layer, in combination with the trace width. The purpose is to demonstrate our technique by means of simulation results that are useful for the highspeed digital designer.

The characteristic impedance of a symmetrical configuration is in reality more often used in the design of transmission line structures than separate values of the capacitance, conductance, inductance, and resistance, because it directly determines the modal reflection coefficients at both ends of the line, depending on the source and load impedance. For a symmetrical structure with two signal lines and a reference conductor, the differential impedance is defined as twice the odd mode impedance, or hence [2],

$$
Z_{\text {diff }}=2 \sqrt{\frac{j \omega\left(L_{s}-L_{m}\right)+\left(R_{s}-R_{m}\right)}{j \omega\left(C_{s}-C_{m}\right)+\left(G_{s}-G_{m}\right)}}
$$

with the subscript $s$ denoting the diagonal elements of the $2 \times 2$ circuit matrices, $m$ the non-diagonal elements, and $C, G, L$, and $R$, respectively, the capacitance, conductance, inductance and resistance elements. Note that $C_{m}$ and $G_{m}$ are always negative while $L_{m}$ and $R_{m}$ are positive. The capacitance and conductance matrices are found with the quasi-static technique described in Section II and III. The 


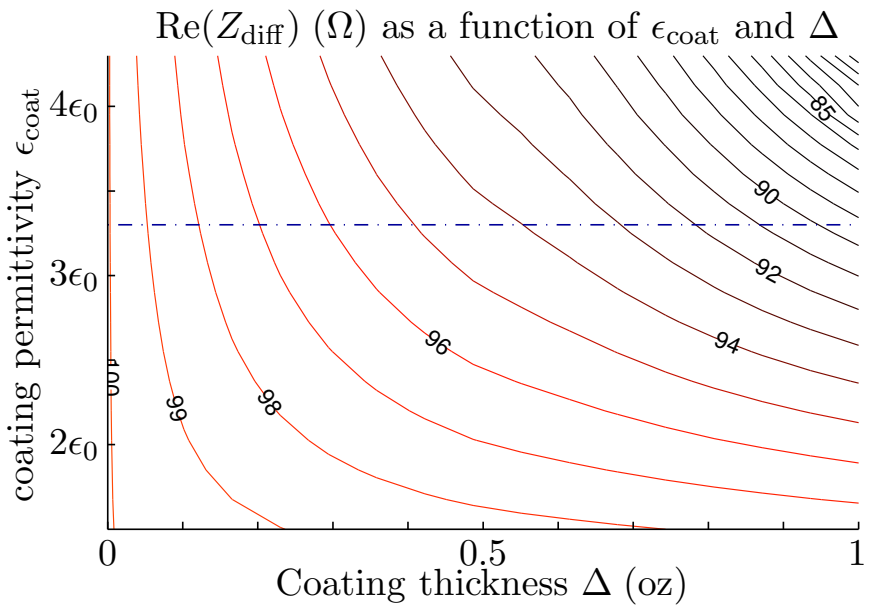

Fig. 8: $\operatorname{Re}\left(Z_{\text {diff }}\right)(\Omega)$ for the structure of Fig. 4, with $w=$ 6.56 mils and at $f=50 \mathrm{GHz}$, for a variable coating permittivity $\epsilon_{\text {coat }}$ and thickness $\Delta$. The dash-dot line $\left(\epsilon_{\text {coat }}=3.3 \epsilon_{0}\right)$ corresponds to the $50 \mathrm{GHz}$ curve of Fig. 7.

resistance and inductance matrices are determined accurately with the technique described in [1], up to high skin effect frequencies.

Some useful information concerning differential pair interconnects from the viewpoint of the designer can be found in [17] and a similar geometry is used here, as shown in Fig. 4. The values of material and geometry parameters are given in the caption, apart from the spacing $s$ between the traces, the coating thickness $\Delta$ and the coating permittivity $\epsilon_{\text {coat }}$, which are varied in the following simulations. Note that the metal thickness is expressed in ounce ('oz'), as is common practice amongst designers $(1 \mathrm{oz}=34.8 \mu \mathrm{m})$. The other dimensions are expressed in 'mils' $(1 \mathrm{mil}=25.4 \mu \mathrm{m})$, following [17]. Furthermore, the total width of the simulated structure is $W=140$ mils, such that the end effect of the finite substrate has a negligible influence on the differential behavior of the line. The coating thickness $\Delta$ is assumed constant along the surface (as is indicated for the right conductor in Fig. 4). The Robin eigenfunction technique described in Sections II and III is applied by modeling the coating as a sequence of 9 connected rectangular dielectric blocks. Fig. 6 shows the 13 rectangular simulation blocks that have been used: 9 passivation blocks, 2 conductor blocks, a dielectric substrate and a ground plane block.

The geometry that we will use as the baseline throughout the different simulations, is the structure as shown in Fig. 4, with $w=6.56$ mils, $\epsilon_{\text {coat }}=3.3 \epsilon_{0}$, and a varying coating thickness $\Delta$. As seen from Fig. 7, the dimensions are chosen such, that its high-frequency differential impedance $Z_{\text {diff }}$ becomes $100 \Omega$ in the case without coating $(\Delta=0)$. The overall behavior is as expected, with a lower impedance as the coating thickness $\Delta$ increases.

In a following simulation, in order to find out how $Z_{\text {diff }}$ depends on the coating permittivity $\epsilon_{\text {coat }}$, the frequency is fixed at $50 \mathrm{GHz}$, and $\epsilon_{\text {coat }}$ is swept from $1.5 \epsilon_{0}$ tot $4.3 \epsilon_{0}$, with the result shown in Fig. 8. The dash-dot line shows the result for $\epsilon_{\text {coat }}=3.3 \epsilon_{0}$, which corresponds to the dash-dot curve at

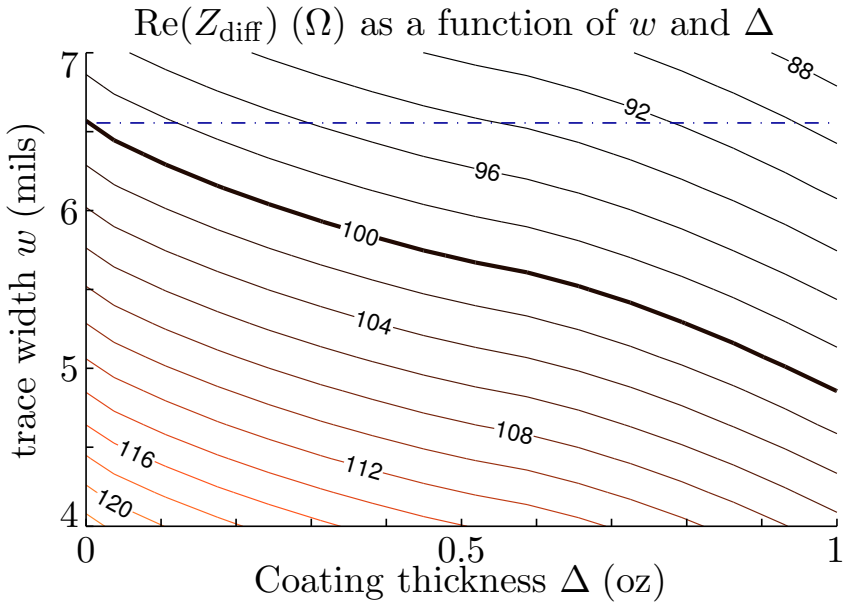

Fig. 9: $\operatorname{Re}\left(Z_{\text {diff }}\right)(\Omega)$ for the structure of Fig. 4, with $\epsilon_{\text {coat }}=$ $3.3 \epsilon_{0}$ and at $f=50 \mathrm{GHz}$, for a variable trace width $w$ and thickness $\Delta$. The dash-dot line corresponds to the dash-dot lines in Fig. 7 and 8.

$50 \mathrm{GHz}$ in Fig. 7. The results displayed in Fig. 8 can be easily interpreted. For an increasing coating thickness, the impedance decreases faster for a higher coating permittivity.

The question is, how the geometry should be modified to compensate for the effect of the coating. In the following experiment, we will reduce the trace width $w$, keeping the spacing between the traces constant. The resulting $Z_{\text {diff }}$ is shown in Fig. 9 as a function of $w$ and $\Delta$. As a reference for the reader, the dash-dot line again corresponds to the result for the baseline geometry, already displayed in Fig. 7 and 8. The bold line shows the required compensation of $w$ as a function of the coating thickness, in order to maintain a $100 \Omega$ differential impedance.

\section{CONCLUSION}

This paper presents a new technique for fast capacitance calculations, based on the Robin eigenmode expansion of the electric potential in homogeneous rectangular dielectric subregions. A careful comparison with the Dirichlet-to-Neumann approach shows that the new technique performs better both in terms of accuracy and efficiency. As a result, challenging configurations with very thin dielectric layers can be dealt with. This is demonstrated with the analysis of a differential microstrip interconnect, coated with a dielectric passivation layer.

\section{ACKNOWLEDGMENT}

The authors would like to thank dr. Bart Baekelandt for his useful advice on some practical aspects of high-speed interconnect design.

\section{REFERENCES}

[1] T. Demeester and D. De Zutter, "Quasi-TM transmission line parameters of coupled lossy lines based on the Dirichlet to Neumann boundary operator," IEEE Trans. Microw. Theory Tech., vol. 56, no. 7, pp. 16491660, Jul. 2008. 
[2] F. Olyslager, Electromagnetic Waveguides and Transmission Lines. Oxford, U.K.: Oxford University Press Inc., 1999.

[3] E. Denlinger, "A frequency dependent solution for microstrip transmission lines," IEEE Trans. Microw. Theory Tech., vol. 19, no. 1, pp. $30-$ 39, Jan. 1971.

[4] C. Wei, R. Barrington, J. Mautz, and T. Sarkar, "Multiconductor transmission lines in multilayered dielectric media," IEEE Trans. Microw. Theory Tech., vol. 32, no. 4, pp. 439 - 450, Apr. 1984.

[5] W. Delbare and D. De Zutter, "Space-domain green's function approach to the capacitance calculation of multiconductor lines in multilayered dielectrics with improved surface charge modeling," IEEE Trans. Microw. Theory Tech., vol. 37, no. 10, pp. $1562-1568$, Oct. 1989.

[6] F. Olyslager, N. Faché, and D. De Zutter, "New fast and accurate line parameter calculation of general multiconductor transmission lines in multilayered media," IEEE Trans. Microw. Theory Tech., vol. 39, no. 6 , pp. $901-909$, jun 1991.

[7] K. S. Oh, D. Kuznetsov, and J. Schutt-Aine, "Capacitance computations in a multilayered dielectric medium using closed-form spatial Green's functions," IEEE Trans. Microw. Theory Tech., vol. 42, no. 8, pp. 1443 -1453 , Aug. 1994.

[8] J. Bernal, F. Medina, and M. Horno, "Quick quasi-tem analysis of multiconductor transmission lines with rectangular cross section," Microwave Theory and Techniques, IEEE Transactions on, vol. 45, no. 9, pp. 1619 -1626 , Sep. 1997.

[9] W. Shu and S. Xu, "Capacitance extraction of multiconductor transmission lines in multilayered dielectric media using numerical green's function," in Antennas and Propagation Society International Symposium, 2003. IEEE, vol. 1, Jun. 2003, pp. 761 - 764 vol.1.

[10] L. Shujing and Z. Hanqing, "An efficient algorithm for the parameter extraction of multiconductor transmission lines in multilayer dielectric media," in Antennas and Propagation Society International Symposium, 2005 IEEE, vol. 3A, Jul. 2005, pp. 228 - 231 vol. 3A.

[11] S. Musa and M. Sadiku, "Application of the finite element method in calculating the capacitance and inductance of multiconductor transmission lines," in Southeastcon, IEEE, Apr. 2008, pp. 300 -304.

[12] J. Van Bladel, Electromagnetic Fields, 2nd ed. The IEEE Press Series on Electromagnetic Wave Theory, Wiley - Interscience, 2007.

[13] P. Kok, F. Olyslager, J. Van Hese, L. Van Hawwermeiren, M. Botte, and D. De Zutter, "Quasi-TEM modelling of two and three dimensional highspeed interconnection structures," in Proc. International Electronics Packaging Conference (IEPS'90), Sep. 1990, pp. 822-826.

[14] T. Demeester and D. De Zutter, "Construction and applications of the Dirichlet to Neumann operator in transmission line modeling," J. Elec. Eng. Comp. Sci., vol. 17, no. 3, pp. 205-216, Nov. 2009.

[15] - "Construction of the Dirichlet to Neumann boundary operator for triangles and applications in the analysis of polygonal conductors," IEEE Trans. Microw. Theory Tech., vol. 58, no. 1, pp. 116-127, Jan. 2010.

[16] H. Johnson, "Passivation and solder mask," Electronics Design, Strategy, News (EDN), Jun. 2002. [Online]. Available: http://www.sigcon.com/Pubs/edn/PassivationandSolderMask.htm

[17] — , "Differential trace impedance," High-Speed Digital Design online newsletter, vol. 5, no. 2, Jan. 2002. [Online]. Available: http://www.sigcon.com/Pubs/news/5_2.htm

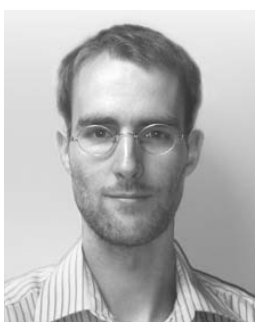

Thomas Demeester was born in 1982 in Ghent, Belgium. He received his M. Sc. Degree in electrical engineering from Ghent University in 2005, after a one-year period at ETH Zürich for his master thesis in the field of time-domain electromagnetics. In 2009 he obtained the Ph.D. degree in electrical engineering, as a Research Fellow of the Fund for Scientific Research, Flanders. His research ranges from electromagnetic field calculations in the presence of highly lossy media and the development of transmission line models for interconnects, to machine learning techniques for optimization and search methods.

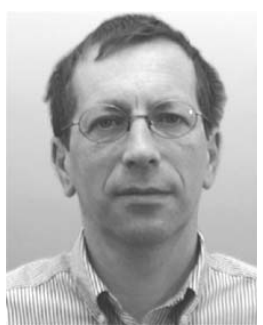

Daniël De Zutter was born in 1953. He received his M. Sc. Degree in electrical engineering from Ghent University in 1976. From 1976 to 1984 he was a research and teaching assistant at the same university. In 1981 he obtained a Ph.D. degree and in 1984 he completed a thesis leading to a degree equivalent to the French Aggrégation or the German Habilitation. From 1984 to 1996 he was with the National Fund for Scientific Research of Belgium. $\mathrm{He}$ is now a full professor of electromagnetics. Most of his earlier scientific work dealt with the electrodynamics of moving media. His research now focusses on all aspects of circuit and electromagnetic modelling of high-speed and high-frequency interconnections, packaging, on-chip interconnect and on numerical solutions of Maxwell's equations. As author or co-author he has contributed to more than 200 international journal papers. In 2000 he was elected to the grade of Fellow of the IEEE. He served as dean of the Faculty of Engineering of Ghent University and as associate editor for the MTT-Transactions. At present he is the head of the Department of Information Technology of Ghent University. 